\title{
Five Reasons for the Failure to Diagnose Aldosterone Excess in Hypertension
}

\author{
Authors \\ George P. Piaditis ${ }^{1}$, Gregory Kaltsas ${ }^{2}$, Athina Markou ${ }^{1}$, George P. Chrousos ${ }^{3}$
}

\begin{abstract}
Affiliations
1 Department of Endocrinology and Diabetes Center,

“G. Gennimatas" General Hospital, Athens, Greece

2 Department of Pathophysiology, National University of Athens, Athens, Greece

3 University Research Institute of Maternal and Child Health and Precision Medicine, National and Kapodistrian University of Athens, Medical School, Athens, Greece
\end{abstract}

\section{Key words}

primary aldosteronism, hypertension, ACTH - adrenal, aldosterone to renin ratio

received 28.05.2020

accepted after revision $\quad 05.08 .2020$

published online $\quad 03.09 .2020$

\section{Bibliography}

Horm Metab Res 2020; 52: 827-833

DOI 10.1055/a-1236-4869

ISSN 0018-5043

(C) 2020. Thieme. All rights reserved. Georg Thieme Verlag KG, Rüdigerstraße 14, 70469 Stuttgart, Germany

\author{
Correspondence \\ Dr. George P. Piaditis \\ Department of Endocrinology and Diabetes Center \\ “G. Gennimatas" General Hospital \\ 154 Mesogion Avenue \\ 11527 Athens \\ Greece \\ Tel.: + 30 6977218429, Fax: +302107979140 \\ edk-pgna@otenet.gr
}

\begin{abstract}
Primary hyperaldosteronism (PA) is a well-known cause of hypertension although its exact prevalence amongst patients with apparent essential hypertension has been a matter of debate. A number of recent studies have suggested that mild forms of PA may be relatively common taking into consideration factors that were previously either overestimated or ignored when developing diagnostic tests of PA and when applying these tests into normotensive individuals. The performance characteristics and diagnostic accuracy of such tests are substantially increased when the adrenocorticotrophin effect, inappropriate potassium levels and their application in carefully selected normotensive individuals are considered. In the present review, we critically analyze these issues and provide evidence that several, particularly mild, forms of PA can be effectively identified exhibiting potentially important clinical implications.
\end{abstract}

\section{Introduction}

Primary aldosteronism (PA) was first described by J. W. Conn in 1955 [1]. Since then it has been considered as the most common and mostly treatable cause of endocrine hypertension [2]. PA is caused by autonomous aldosterone production from the adrenal glands, independently of the renin-angiotensin system (RAS), mainly by an adrenal adenoma or bilateral adrenocortical hyperplasia $[3,4]$. Aldosterone exerts its predominant genomic actions in the epithelial cells of the distal convoluted tubules of the kidney [5], after binding the cytoplasmic/nuclear mineralocorticoid receptor (MR), causing retention of sodium and water. Through this mechanism, as well as by activating the sympathetic nervous system directly, aldosterone excess causes hypertension. In addition, aldosterone excess itself in the context of excess sodium, promotes chronic tissue inflammation, causing fibrosis in vital organs, such as blood vessels, heart and kidney, and leading to cardiac arrhythmias, myocardial infarction, stroke, and renal failure [6-14].

The current standard practice for the diagnosis of PA is to initially apply a screening test, using the calculation of basal aldosterone-to-renin ratio (ARR), followed by a diagnostic test to confirm or exclude autonomous aldosterone secretion, such as the oral sodium loading test, saline infusion test (SIT), fludrocortisone suppression test (FST), or captopril challenge test [15]. Using current methodology, the estimated prevalence of PA ranges from 4.6 to $16.6 \%$ [16-21]. However, recent studies from our department, using the modified saline loading diagnostic tests FDST (fludrocortisone-dexamethasone suppression test) or D-SIT (post dexamethasone saline infusion test), have shown that the prevalence of PA in unselected patients with presumed idiopathic hypertension is approximately $30 \%[22,23]$. This discrepancy from earlier studies 
is due to the use of different diagnostic methods, the selection criteria of the study population and the severity of hypertension of the patients included in different studies [16-19]. Furthermore, in daily practice besides the different assay methods used there also exists a wide range of different cut-offs of ARR, leading to the demand of Endocrine Society Guideline for 'individual' values for PAC, renin concentration/PRA, and ARR of laboratories. Despite this general consideration, the real underlying reasons for such a large discrepancy regarding the prevalence of PA have never been searched in a systematic way and remain unclear. This long-lasting challenge led us to an extensive scrutiny of the international literature. To our surprise, there were at least five different factors leading to a significant underestimation of PA prevalence in hypertension, which were either ignored or underestimated.

\section{Unaccounted Adrenocorticotrophin (ACTH) Effect on Aldosterone Secretion}

It is generally accepted that the main regulators of aldosterone secretion are the RAS and serum potassium levels, whereas ACTH is thought to exert a relatively minor effect. However, in a recent study we identified a group of hypertensive patients with apparent essential hypertension without PA (FDST-negative) that exhibited an exaggerated acute aldosterone response to ultra-low ACTH $(0.03 \mu \mathrm{g})$ stimulation or a treadmill test, three times higher compared to a group of normotensive controls, whereas cortisol response did not differ between the two groups [24]. Similar results have been reported in previous studies, where the effect of graded intensities treadmill exercise (corresponding to 50,70 , and $90 \%$ of maximum oxygen uptake, $\mathrm{VO}_{2 \max }$ ) on aldosterone secretion was examined $[25,26]$. A significant aldosterone response was observed even with mild exercise $\left(50 \% \mathrm{VO}_{2 \max }\right)$, while the maximum response was observed with moderate exercise $\left(70 \% \mathrm{VO}_{2 \max }\right)$. In contrast, cortisol levels remained unchanged at mild exercise, while the maximum response was observed with severe exercise $(90 \%$ $\left.\mathrm{VO}_{2 \max }\right)$. These results suggest that the adrenal cortex is more ready to secrete aldosterone acutely than cortisol in response to ACTH during physical stress.

In addition, many other in vivo and in vitro studies show that ACTH may induce a short-lasting acute and a long-lasting effect on aldosterone secretion [27]. There is evidence showing that the aldosterone response to chronic infusion of ACTH is directly related to the pattern of ACTH infusion [28]. Continuous i. v. ACTH infusion leads to a sustained cortisol secretion, but a short-lasting aldosterone response, which declines to pre-stimulation levels by $48 \mathrm{~h}$. In contrast, pulsatile infusion of ACTH, which resembles the normal pattern of ACTH secretion, led to a prolonged aldosterone response, which was maintained up to $72 \mathrm{~h}$. As a consequence, a major question is raised regarding the physiologic significance of this acute aldosterone response to ACTH stimulation. Although a clear answer is not available, there are reports showing that aldosterone via genomic MR-mediated and/or non-genomic MR-independent action, may affect the function of many tissues and organs. Thus, it may modify the expression of several genes of the cardiovascular system [29], which mediate different biochemical phenomena, enhances the activity of the sympathetic nervous system [30] and stimulates the activity of $\mathrm{H}^{+}$-ATPase in the acid-sec- retary intercalated cells of isolated outer medullary collecting ducts and epithelial $\mathrm{Na}^{+}$channels $(\mathrm{ENaC})$ in the rat kidney [31].

These data show that ACTH, even under very mild stressful conditions, as these encountered during daily physical activities or psychological events, is a significant natural stimulator of aldosterone secretion. Therefore, ACTH should be considered in the development of any provocative test aiming to diagnose autonomous aldosterone secretion. Unfortunately, in all currently employed PA diagnostic tests, ACTH has been largely ignored, targeted to cause suppression (saline loading tests) or inhibition (captopril test) of the RAS, as it is considered as the main strong stimulator of aldosterone secretion. However, in our opinion, this represents a long-standing systematic error, granted that the fluctuating ACTH levels may shift the estimated aldosterone normal cut-offs by all these tests upwards, leading to under-estimation of PA prevalence in hypertension. This is supported by the results of recent studies, where we used either the FDST, where $2 \mathrm{mg}$ of dexamethasone were given at mid-night of the 4th day of the test [22], or the post $\mathrm{D}$-SIT, where the low dose dexamethasone suppression test was followed by $2 \mathrm{Lt}$ normal saline infusion over $4 \mathrm{~h}$ [23]. In both cases, a much higher prevalence of PA in hypertension was observed (30 and $24 \%$, respectively) compared to the published figures.

\section{Basal ARR: A widely used screening test for PA with high sensitivity. An untested long- standing hypothesis}

The ARR was initially described by Hiramatsu et al. in 1981 [32] and since then has been the most widely recommended first line screening test for PA. The standard procedure recommended by the Endocrine Society Clinical Practice Guideline [15] is to initially utilize the ARR ratio as a screening test aiming to identify a sub-population of hypertensives with suspected AAS and then to perform a diagnostic test to confirm or exclude the diagnosis of PA. This practice should be ideal if we considered the ARR a test of very high sensitivity, which, however, has never been validated.

The only appropriate approach to evaluate the performance characteristics of the ARR screening test is to identify all the PA cases included in an unselected population of hypertensives using a highly sensitive diagnostic test applied to all participants of the study in advance, not after the use of ARR and, therefore, regardless of the results of ARR screening. Although, neither of the available confirmatory tests is considered as a 'gold standard test' to discriminate PA from essential hypertensive patients reliably, there is only a single study that has adopted this approach of using a community-based sample of 118 Caucasian hypertensive patients [33]. The estimated specificity of ARR was found to vary from 71 to $84 \%$, with a concomitant sensitivity ranging from 73 to $93 \%$, whereas the prevalence of PA was found to be $13 \%$. The major limitation of this study was that the diagnosis of PA was made by measuring only 24-h urinary aldosterone excretion, whereas the control group was not tested for the presence of adrenal adenomas, while the ACTH effect on aldosterone secretion was not considered.

However, in a recently published study where the FDST, a diagnostic test with very high sensitivity and specificity, was performed in all hypertensives, rather than those identified by the ARR ratio, the number of hypertensives who had PA based on an elevated 
basal ARR ratio was significantly lower than that identified by the FDST (43/324 vs. 94/324) [34]. The basal ARR ratio as a screening test had, at $67.5 \mathrm{pmol} / \mathrm{mU}$, an estimated sensitivity of $35 \%$ and specificity of $95 \%$, which were, respectively, much lower and much higher than previously appreciated [35]. The results of this study clearly showed that the use of basal ARR as a screening test is one of the major causes leading to significant underestimation of PA prevalence in hypertension, as it may recognize only the minority of PA patients, mainly those with more severe disease, leaving undiagnosed the milder cases. An impressive improvement in the performance characteristics of the ARR ratio was observed when the FDST was used, as the estimated sensitivity and specificity of the post-FDST ARR ratio were 100 and $97.58 \%$, respectively $[22,36]$. This suggests that suppression of the stimulatory effect of both ACTH and renin on aldosterone secretion is required to establish the appropriate cut-offs and identify with high sensitivity and specificity, cases of subclinical PA among hypertensives.

\section{Normotensive Controls Recruitment}

The recruitment of appropriate controls is essential and necessary to define reliable normal cut-offs for any diagnostic test. Unfortunately, this does not seem to apply to the determination of the normal cut-offs of either the screening or the diagnostic tests for PA, as these cut-offs in all published studies have been obtained from apparently normal controls recruited using clinical criteria (normal blood pressure and serum potassium levels) alone, without imaging studies of the adrenal glands. However, approximately 5-10\% of patients undergoing imaging of the abdomen for non-adrenal related diseases harbor incidentally detected, clinically inapparent adrenal masses [37]. The great majority of these masses are benign adrenal adenomas, which in a significant proportion exhibit autonomous aldosterone secretion $[23,36]$.

A recent study revealed that 7 out of 79 (8.8\%) normotensive individuals who served as controls had a single adrenal adenoma on abdominal computerized tomography (CT) [22], whereas four of them had also PA, as their post-FDST aldosterone levels were significantly higher, ranging from 82 to $168 \mathrm{pmol} / \mathrm{l}$, compared with the cut-off level of $74 \mathrm{pmol} / \mathrm{l}$ obtained from individuals with normal adrenal imaging [22]. In another recent study, where the pattern of aldosterone secretion in patients with adrenal incidentalomas with and without arterial hypertension was tested, we showed that $12 \%$ of the patients with normal blood pressure exhibited significant autonomous aldosterone secretion [36]. Furthermore, there are strong pieces of evidence suggesting that PA may also be present in pre-hypertension, in normotensive individuals without adrenal adenomas, which is extensively discussed in a recent review [16]. However, it is worth to mention a recently published prospective study, where 13 from 100 recruited normotensive controls with normal adrenal imaging had PA by their post-FDST aldosterone levels and ARR, which ranged from 100 to $360 \mathrm{pmol} / \mathrm{l}$ and from 30 to $85 \mathrm{pmol} / \mathrm{mU}$, respectively ( $>$ Fig. 1), values much higher than those of 67 controls without PA, who remained normotensive after a follow-up period of 7 years [38]. It was interesting that 11 (85\%) of these 13 controls with PA developed hypertension compared with only 20 of the 87 (23\%), who had normal aldosterone suppression to FDST (odds ratio: 18.42, $95 \%$ confidence interval:
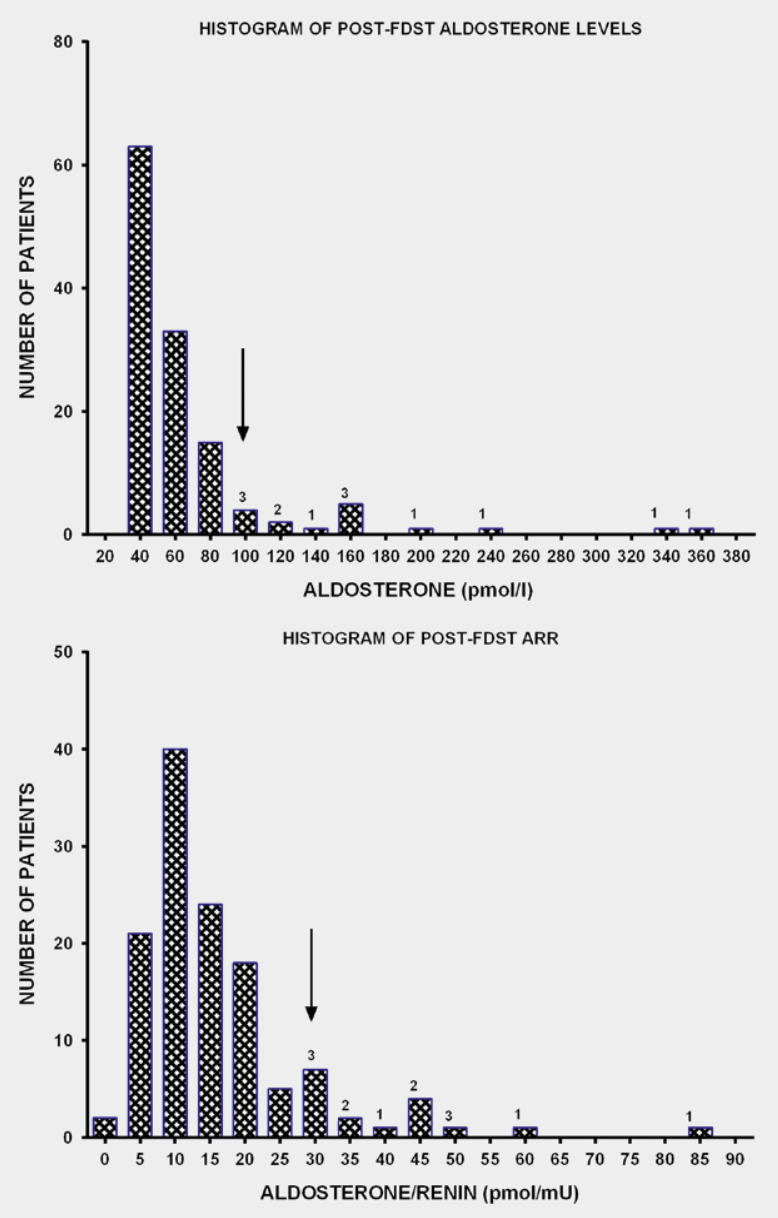

- Fig. 1 Histograms of post-FDST aldosterone levels and ARR in 100 normotensive controls with normal imaging of adrenal glands. Thirteen controls had both post-FDST aldosterone levels and ARR compatible with autonomous aldosterone secretion.

3.76-90.10, p<0.0001). Similar results were reported in recent studies by Baudrant et al. and Brown et al. where autonomous aldosterone secretion was detected in 14 and $11 \%$ respectively of normotensive populations $[39,40]$. These data suggest the adoption of strict selection criteria for any control group used to define normal cut-offs for PA, by recruiting individuals with long-standing normal BP and normal adrenal imaging. Search of the literature revealed that such criteria have never been adopted by any of the published studies so far. Thus, it is quite possible that the populations regarded as 'normal controls' in these studies may include normotensive PA participants with or without adrenal adenomas, who therefore may have shifted the aldosterone and ARR normal cut-offs upwards, leading finally to underestimation of the overall prevalence of PA in patients with hypertension.

A potential issue on using these tests could arise in populations with substantially low renin levels such as in certain Afro-American ethnicities. In such cases adequately selected controls may be need to define the range of upper normal limits that need to be considered. 


\section{Hypokalemia-induced Saline Loading Tests}

It is well known that potassium is a natural regulator of aldosterone secretion, even under small changes of its serum levels within the normal range [41,42], whereas hypokalemia is a strong inhibitor of aldosterone synthesis mainly by a direct action on adrenal cortex [43]. It is, therefore, obvious that serum $\mathrm{K}^{+}$levels can directly affect the diagnostic accuracy of any available screening or diagnostic test for PA. That is why, it is fundamental to recommend that the serum $\mathrm{K}^{+}$levels must be kept strictly within the normal limits during these tests $[4,15]$. This concerns in particular all diagnostic tests targeting suppression of RAS by saline loading (FST, FDST, SIT, $\mathrm{D}-\mathrm{SIT}$ ), during which a significant reduction in serum $\mathrm{K}^{+}$levels is often observed, despite simultaneous oral potassium administration. Although it is well characterized, the effect of serum $\mathrm{K}^{+}$on the diagnostic accuracy of saline loading tests has never been validated, mainly because of the lack of alternative diagnostic tests for PA with high diagnostic accuracy to compare with. However, this has been validated in only a single recent study, where an overnight, of high sensitivity and specificity, screening and diagnostic test for PA based on pharmaceutical blockade of ACTH and RAS with dexamethasone, captopril and valsartan (DCVT) [44], was compared with the FDST which, in contrast, is based on suppression of RAS by oral sodium loading. This study showed that the effect of FDST and DCVT on serum $\mathrm{K}^{+}$levels was of particular importance. FDST induced a significant decrease in serum $\mathrm{K}^{+}$levels in both essential hypertensive (EH) patients and PA patients. By contrast, DCVT induced a small but significant increase in serum $\mathrm{K}^{+}$ levels, within the normal range, in EH patients, whereas the test did not affect serum $\mathrm{K}^{+}$levels in patients with PA ( $\bullet$ Fig. 2). FDST failed to diagnose PA in four patients, who were considered to have the disease, as they presented with treatment-resistance hypertension under therapy with two to three antihypertensive drugs, spontaneous hypokalemia, suppressed renin levels and kaliuresis, while all of them normalized their blood pressure after administration of spironolactone or eplerenone. In contrast, DCVT diagnosed severe
PA in all four patients. It is interesting that only these four patients among the tested hypertensives completed the FDST with significant hypokalemia ( $\mathrm{K}^{+}$levels: $2.5-3.1 \mathrm{mEq} / \mathrm{I}$ ) and, therefore, low potassium could be implicated for the FDST failure to diagnose PA in these four cases. These results show that hypokalemia may induce severe blockade of aldosterone biosynthesis, affecting the sensitivity of saline loading tests.

\section{Validation of diagnostic test for PA using aldosterone levels alone}

The current method to evaluate the diagnostic tests of PA is based on the estimation of the post-test aldosterone levels alone, without consideration of the concomitant renin levels. This is a long-standing erroneous choice granted that aldosterone secretion is directly depended on serum renin levels. This concerns all the diagnostic tests for PA, but especially those based on extracellular volume expansion-mediated RAS suppression by sodium loading either intravenously (SIT) or orally (FST, FDST). In the case of SIT, sodium is administered i. v. as $0.9 \%$ normal saline ( 2 liters over $4 \mathrm{~h}$ ) and, therefore, is expected to expand extracellular volume and suppress the post-SIT aldosterone levels. However, the crucial question raised is whether the amount of infused normal saline is high enough to induce adequate expansion of extracellular volume sufficient to achieve complete suppression of RAS. The only reliable answer to this question should be given by measuring serum renin, which, however, is not the current common practice for the evaluation of diagnostic of PA tests. Therefore, by measuring the posttest aldosterone levels alone we cannot be sure that SIT can achieve adequate RAS suppression either in normal populations used as controls to define the normal cut-offs or to hypertensive patients with suspected PA. Inadequate RAS suppression in controls may shift the estimated normal cut-offs of post-test aldosterone upwards affecting the sensitivity of the test, while in hypertensives it
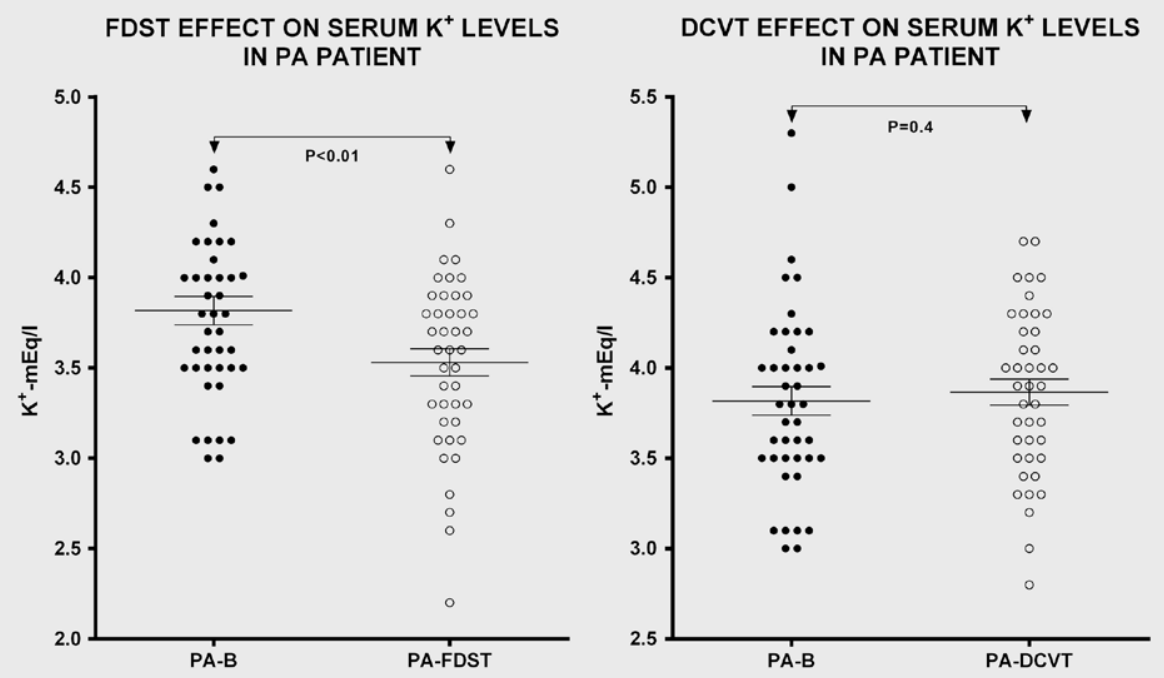

-Fig. 2 FDST and DCVT effect on serum $\mathrm{K}^{+}$levels in patients with PA. FDST induced a significant decrease, whereas DCVT did not have any effect in serum $\mathrm{K}^{+}$levels in patients and PA. 
may cause falsely elevated post-test aldosterone levels affecting its specificity.

This was shown in a recent study, where both the SIT and D-SIT were used to define the prevalence of PA in patients with a single adrenal incidentaloma (SAI) [23]. In this study we found that both SIT and D-SIT failed to suppress renin levels in 19 (23\%) and 23 (27\%) ( $\triangleright$ Fig. 3) out of 83 controls respectively, leading to unsuppressed aldosterone levels, while their port-SIT and post-D-SIT ARR was within the normal limits (false positive results). Interestingly, their $24 \mathrm{~h}$ urinary sodium was very low, ranging from $50-88 \mathrm{mmol} / 24 \mathrm{~h}$, suggesting that the latter is related to long-standing sodium intake restriction, as the consumption of salt has been implicated in the development of hypertension internationally. However, the unsuppressed aldosterone levels in a significant proportion of controls, by definition, shift its upper normal limit (UNL) upwards, affecting seriously the sensitivity of the tests. Using the mean + 2SD, the estimated post-SIT aldosterone UNL was 158.30 pmo/I, much higher compared to post-D-SIT aldosterone UNL, estimated at $66.67 \mathrm{pmol} / \mathrm{l}$. Similar results were obtained with ARR, as the estimated post saline infusion UNL of ARR was 30.68 and $9.60 \mathrm{pmol} / \mathrm{mU}$ for SIT and D-SIT respectively. The impressive difference between the post-SIT and post-D-SIT aldosterone and ARR UNL should be ACTH related, as the D-SIT was performed under completely suppressed ACTH levels, while the SIT with ACTH levels freely fluctuated, whereas the post saline infusion renin levels did not differ between the two tests (> Fig. 3).

Interestingly, after applying each of these newly defined cutoffs separately (the post-D-SIT aldosterone level or the post-D-SITARR ratio), 17 (20.48\%) patients with SAl exhibited an abnormality in only one of the two cut-offs. Ten (12.05\%) patients had elevated post-D-SIT aldosterone levels and normal post-D-SIT ARR, whereas 7 (8.44\%) patients had an elevated post-D-SIT ARR ratio and normal post-D-SIT aldosterone levels. These patients would have erroneously been considered to have PA if either the post-SIT aldosterone levels or ARR alone were employed (false-positive results). The above results show clearly that neither post-SIT and post-D-SIT aldosterone nor ARR can be used separately as a reliable index to validate the diagnostic saline loading tests of PA. Using
CONTROLS

RENIN LEVELS: POST-SIT AND POST-DST
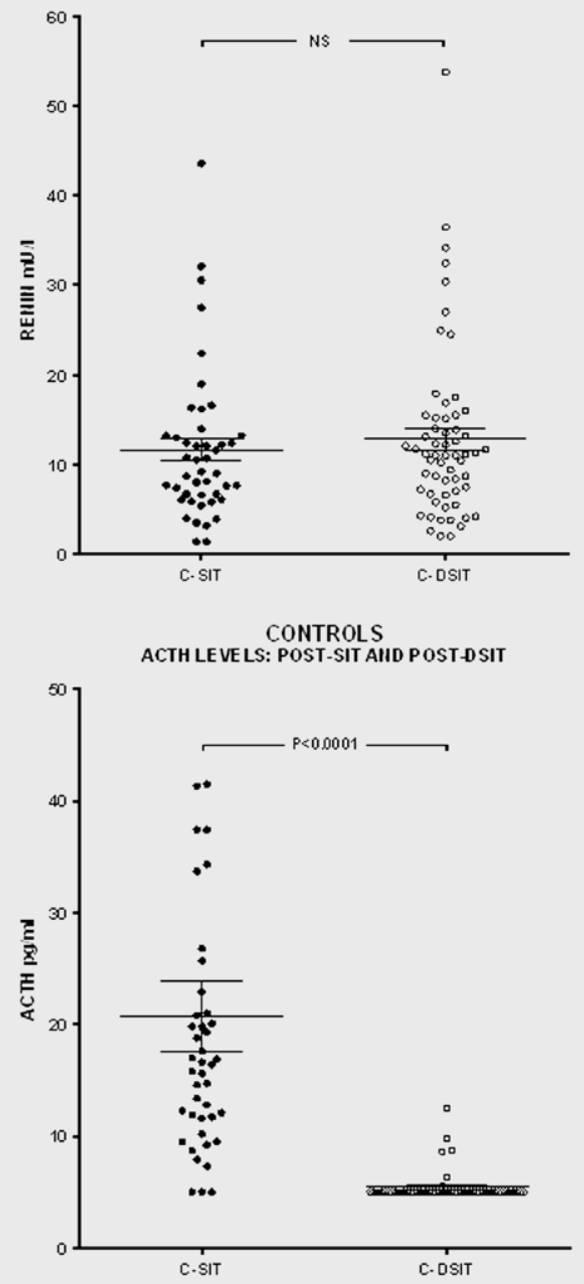

PATENTS

RENIN LEVELS: POST-STT AND POST DST

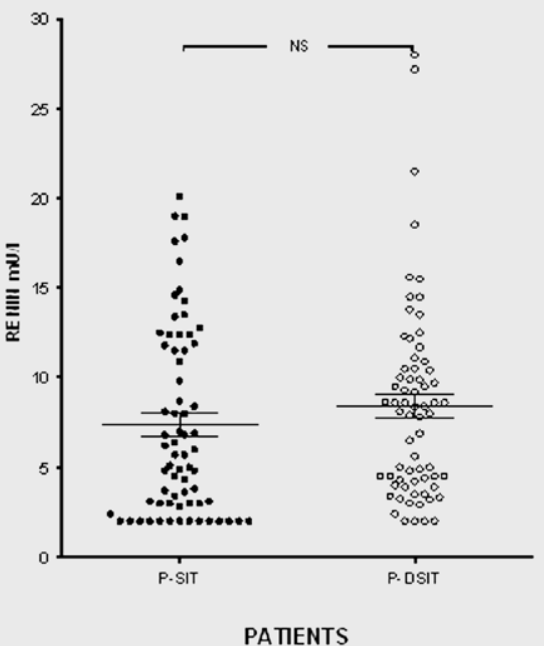

ACTH LEVELS: POS T-SIT AIID POSTDSIT

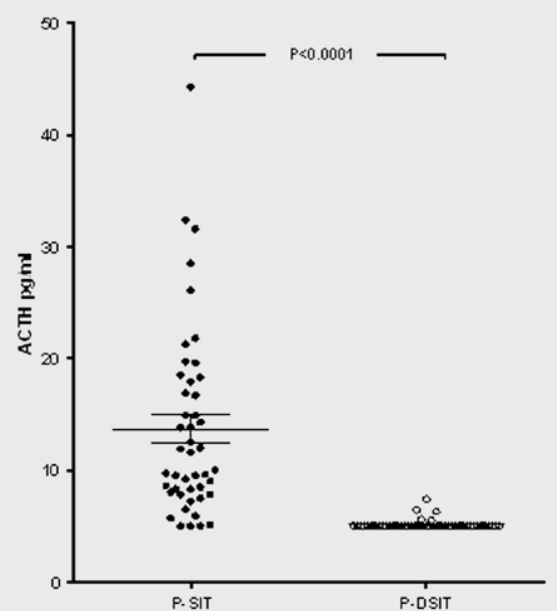

- Fig. 3 Post-SIT and post-D-SIT renin and ACTH levels in normotensive controls and hypertensive patients. C: Controls, P: Patients. 
the UNL of basal ARR $(50.80 \mathrm{pmol} / \mathrm{mU})$ and the post-SIT aldosterone levels (158.30 pmol/mU), which is the currently employed diagnostic procedure for PA, the estimated prevalence of PA was $13.40 \%$, which was much lower compared to PA prevalence (29\%) estimated by simultaneous consideration of post-D-SIT UNL of aldosterone $(66.70 \mathrm{pmol} / \mathrm{I})$ and ARR $(9.60 \mathrm{pmol} / \mathrm{mU})$. Therefore, only patients who had both post-D-SIT aldosterone levels and post-DSIT ARR higher than the defined normal cut-offs were considered to have PA.

The above is particularly relevant to the outpatient performance of the oral sodium loading tests FST and FDST, as compliance of both controls and hypertensive patients with the recommended by the protocol daily sodium consumption is imperative. In a recent study, in which the FDST was used to estimate the prevalence of PA in an unselected hypertensive population $[22,34]$, the estimated specificity of post-FDST ARR and aldosterone levels was $96 \%$ and $61 \%$, respectively. This means that about $40 \%$ of the hypertensive patients with EH had non-suppressed post-FDST aldosterone levels (false positive results). This is because the oral saline loading failed to suppress the post-FDST renin levels, whereas another $4 \%$ had falsely elevated post-FDST ARR, because of inappropriate low renin levels. Therefore, the simultaneous consideration of both post-FDST aldosterone and ARR is necessary for a reliable diagnosis of PA.

\section{Summary}

Despite major discoveries and scientific advances in endocrinology, primary aldosteronism and its diagnosis remain challenging. Our diagnostic tools continue to improve, gradually decreasing the term "idiopathic" in front of the name of many nosologic entities. Hypertension is one of them. Our knowledge of physiology and pathophysiology expands, our diagnostic tools become more sensitive and specific and we now are better able to diagnose, prevent and treat. The 5 caveats described above should not hinder our work with primary aldosteronism. We are certain, more will be discovered and corrected as the bright future of our field unfolds and technology makes huge leaps particularly as a number of existing genetic alterations may identify different patients' subgroups. As 5 th century BCE Herakleitos had suggested "If you do not search for the unexpected you are not going to find it".

\section{Conflict of Interest}

The authors declare that they have no conflict of interest.

\section{References}

[1] Conn JW, Louis LH. Primary aldosteronism: A new clinical entity. Trans Assoc Am Physicians 1955; 68: 215-231

[2] Schirpenbach C, Reincke M. Primary aldosteronism: Current knowledge and controversies in Conn's syndrome. Nature clinical practice. Endocrinol Metab 2007; 3: 220-227
[3] Gordon RD, Gomez-Sanchez CE, Hamlet SM et al. Angiotensin-responsive aldosterone-producing adenoma masquerades as idiopathic hyperaldosteronism (IHA: Adrenal hyperplasia) or low renin essential hypertension. J Hypertens (Suppl) 1987; 5: S103-S106

[4] Stowasser M, Gordon RD, Rutherford JC et al. Diagnosis and management of primary aldosteronism. JRAAS 2001; 2: 156-169

[5] Staruschenko A. Regulation of transport in the connecting tubule and cortical collecting duct. Compr Physiol 2012; 2: 1541-1584

[6] Briet M, Schiffrin EL. The role of aldosterone in the metabolic syndrome. Curr Hypertens Rep 2011; 13: 163-172

[7] Rossi GP, Sacchetto A, Pavan E et al. Left ventricular systolic function in primary aldosteronism and hypertension. J Hypertens 1998; 6: 2075-2077

[8] Rossi GP, Di Bello V, Ganzaroli C et al. Excess aldosterone is associated with alterations of myocardial texture in primary aldosteronism. Hypertension 2002; 40: 23-27

[9] Rossi GP, Bernini G, Desideri G et al. Renal damage in primary aldosteronism: Results of the PAPY Study. Hypertension 2006; 48: 232-238

[10] Rossi GP, Sechi LA, Giacchetti G et al. Primary aldosteronism: Cardiovascular, renal and metabolic implications. Trends Endocrinol Metab 2008; 19: 88-90

[11] Fritsch Neves M, Schiffrin EL. Aldosterone: A risk factor for vascular disease. Curr Hypertens Rep 2003; 5: 59-65

[12] Gilbert KC, Brown NJ. Aldosterone and inflammation. Curr Opin Endocrinol Diabetes Obes 2010; 17: 199-204

[13] Carey RM. Aldosterone and cardiovascular disease. Curr Opin Endocrinol Diabetes Obes 2010; 17: 194-198

[14] Huang BS, Leenen FH. Mineralocorticoid actions in the brain and hypertension. Curr Hypertens Rep 2011; 13: 214-220

[15] Funder JW, Carey RM, Mantero F et al. The management of primary aldosteronism: case detection, diagnosis, and treatment: An Endocrine Society Clinical Practice Guideline. J Clin Endocrinol Metab 2016; 101: 1889-1916

[16] Piaditis G, Markou A, Papanastasiou L et al. Progress in aldosteronism: A review of the prevalence of primary aldosteronism in pre-hypertension and hypertension. Eur J Endocrinol 2015; 172: R191-R203

[17] Rossi GP, Bernini G, Caliumi C et al. A prospective study of the prevalence of primary aldosteronism in 1125 hypertensive patients. J Am Col Cardiol 2006; 48: 2293-2300

[18] Stowasser M. Update in primary aldosteronism. J Clin Endocrinol Metab 2009; 94: 3623-3630

[19] Douma S, Petidis K, Doumas M et al. Prevalence of primary hyperaldosteronism in resistant hypertension: A retrospective observational study. Lancet 2008; 371: 1921-1926

[20] Stowasser M, Gordon RD, Gunasekera TG et al. High rate of detection of primary aldosteronism, including surgically treatable forms, after 'non-selective' screening of hypertensive patients. J Hypertens 2003; 21: 2149-2157

[21] Young WF. Primary aldosteronism: renaissance of a syndrome. Clin Endocrinol 2007; 66: 607-618

[22] Gouli A, Kaltsas G, Tzonou A et al. High prevalence of autonomous aldosterone secretion among patients with essential hypertension. Eur J Clin Inves 2011; 41: 1227-1236

[23] Piaditis GP, Kaltsas GA, Androulakis II et al. High prevalence of autonomous cortisol and aldosterone secretion from adrenal adenomas. Clin Endocrinol (Oxf) 2009; 71: 772-778

[24] Markou A, Sertedaki A, Kaltsas G et al. Stress induced aldosterone hyper-secretion in a substantial subset of patients with essential hypertension. J Clin Endocrinol Metab 2015; 100: 2857-2864 
[25] Luger A, Deuster PA, Debolt JE et al. Acute exercise stimulates the renin-angiotensin-aldosterone axis: Adaptive changes in runners. Horm Res 1988; 30: 5-9

[26] Luger A, Deuster PA, Kyle SB et al. Acute hypothalamic-pituitary-adrenal responses to the stress of treadmill exercise. Physiologic adaptations to physical training. N Engl J Med 1987; 316: 1309-1315

[27] El Ghorayeb N, Bourdeau I, Lacroix A. Role of ACTH and other hormones in the regulation of aldosterone production in primary aldosteronism. Front Endocrinol 2016; 7. Article 72

[28] Seely EW, Conlin PR, Brent GA et al. Adrenocorticotropin stimulation of aldosterone: prolonged continuous versus pulsatile infusion. J Clin Endocrinol Metab 1989; 69: 1028-1032

[29] Turchin A, Guo CZ, Adler GK et al. Effect of acute aldosterone administration on gene expression profile in the heart. Endocrinology 2006; 147: 3183-3189

[30] Schmidt BM, Georgens AC, Martin N et al. Interaction of rapid nongenomic cardiovascular aldosterone effects with the adrenergic system. J Clin Endocrinol Metab 2001; 86: 761-767

[31] Winter C, Kampik NB, Vedovelli L et al. Aldosterone stimulates vacuolar $\mathrm{H}^{+}$-ATPase activity in renal acid-secretory intercalated cells mainly via a protein kinase C-dependent pathway. Am J Physiol Cell Physiol 2011; 301: C1251-C1261

[32] Hiramatsu K, Yamada T, Yukimura Y et al. A screening test to identify aldosterone-producing adenoma by measuring plasma renin activity. Results in hypertensive patients. Arch Intern Med 1981; 141: 1589-1593

[33] Schwartz GL. Screening for primary aldosteronism in essential hypertension: Diagnostic accuracy of the ratio of plasma aldosterone concentration to plasma renin activity. Clin Chem 2005; 51: 386-394

[34] Papanastasiou L, Markou A, Pappa T et al. Primary aldosteronism in hypertensive patients: Clinical implications and target therapy. Eur J Clin Invest 2014; 44: 697-706
[35] Montori VM, Young WF. Use of plasma aldosterone concentration to plasma renin ratio as a screening test for primary aldosteronism: A systematic review of the literature. Endocrinol Metab Clin North Am 2002; 31: 619-632

[36] Pappa T, Papanastasiou L, Kaltsas G et al. Pattern of adrenal hormonal secretion in patients with adrenal adenomas: the relevance of aldosterone in arterial hypertension. J Clin Endocrinol Metab 2012; 97: E537-E545

[37] Mansmann G, Lau J, Balk E et al. The clinically inapparent adrenal mass: Update in diagnosis and management. Endocr Rev 2004; 25 : 309-340

[38] Markou A, Pappa T, Kaltsas G et al. Evidence of primary aldosteronism in a predominantly female cohort of normotensive individuals: A very high odds ratio for progression into arterial hypertension. J Clin Endocrinol Metab 2013; 98: 1409-1416

[39] Baudrand R, Guarda F], Fardella C et al. A continuum of renin-independent aldosteronism in normotension. Hypertension 2017; 69: 950-956

[40] Brown JM, Siddiqui M, Calhoun DA et al. The unrecognized prevalence of primary aldosteronism. Ann Inter Med 2020; 173: 10-20

[41] Himathongkam T, Dluhy RG, Williams GH. Potassium-aldosterone-rennin interrelationships. J Clin Endocrinol Metab 1975; 41: 153-159

[42] Bauer JH, Gauntner WC. Effect of potassium chloride on plasma rennin activity and plasma aldosterone during sodium restriction in normal man. Kidney Int 1979; 15: 286-293

[43] Hattangady N, Olala L, Bollag WB et al. Acute and chronic regulation of aldosterone production. Mol Cell Endocrinol 2012; 350: 151-162

[44] Tsiavos V, Markou A, Papanastasiou L et al. A new highly sensitive and specific overnight combined screening and diagnostic test for primary aldosteronism. Eur J Endocrinol 2016; 175: 21-28 